\title{
Effects of Resilience on Impulsivity, Cognition and Depression during Protracted Withdrawal among Chinese Male Methamphetamine Users
}

\section{Siyao Zhou}

Wenzhou Medical University

\section{Wang Ran}

Zhejiang University

Chenhui Peng

Wenzhou Medical University

Siyu Tong

Wenzhou Medical University

\section{Lan Hong}

Wenzhou Medical University

\section{Fangfang Cai}

Wenzhou Medical University

Wei Jin

Wenzhou Medical University

Yile Jiang

Wenzhou Medical University

Mengjia Li

Wenzhou Medical University

Xuanping Wang

Wenzhou Medical University

Mengdan Luo

Wenzhou Medical University

$\mathrm{He} \mathrm{He}$

Wenzhou Medical University

Wei Wang

Wenzhou Medical University

Ke Zhao ( $\nabla$ cocozk1986@163.com )

Wenzhou Medical University 
Keywords: Methamphetamine, resilience, impulsivity, cognitive function, depression

Posted Date: January 12th, 2022

DOI: https://doi.org/10.21203/rs.3.rs-1209591/v1

License: (c) (1) This work is licensed under a Creative Commons Attribution 4.0 International License. Read Full License 


\section{Abstract \\ Background}

Methamphetamine (METH) dependence is a complex and dynamic public health problem. Long-term abuse of METH can increase numerous risks of mental and physical problems. Currently, the methods to reduce METH dependence and improve the withdrawal symptoms are limited and ineffective. Resilience is regarded as an individuals' protective moderator against stress and may play a role in the stress-related mental diseases.

\section{Methods}

One hundred and twelve males with METH dependence were consecutively recruited from Huanglong Compulsory Isolated Detoxification Center between 2019 and 2021. The Connor-Davidson Resilience Scale (CD-RISC), Self-rating depression scale (SDS), Self-rating anxiety scale (SAS), Barratt Impulsiveness Scale-11(BIS-11), and the Repeatable Battery for the Assessment of Neuropsychological Status (Rbans) were used to evaluate resilience, depression, anxiety, impulsivity, and cognition respectively.

\section{Results}

The results showed that high and medium resilience groups showed lower SDS scores than the low resilience group $(p<0.05)$, with high resilience groups showing lower scores using $S A S(p<0.05)$. The high resilience group demonstrated lower cognitive impulsiveness, non-planning impulsiveness, and BIS-11 scores than the low resilience group (all $\mathrm{p}<0.05$ ). Additionally, compared with the low resilience group, attention was better in the medium resilience group, while delayed memory was better in the high resilience group (both $p<0.05$ ). The total scores of Rbans were also higher in the medium and high resilience groups than low resilience group (both $p<0.05$ ).

\section{Conclusions}

This study confirmed a correlation between resilience and impulsivity, cognitive function, and depression. It may suggest a potential role of resilience for individuals during protracted METH withdrawal.

\section{Introduction}

According to 2020 World Drug Report, approximately 27 million people are estimated to have used amphetamines worldwide in the past year. The number of people using amphetamine, especially methamphetamine (METH), is increasing in parts of Asia and North America [1]. METH addiction is a biological psychosocial disorder that remains a global complex and dynamic public health problem [2]. In China, there is an increasing number of individuals addict to METH[3]. Currently, the methods to reduce 
METH dependence, improve the symptoms caused by METH use, and prevent relapse are limited and ineffective[4, 5]. The available methods primarily consist of cognitive- behavioral therapy(CBT), emergency management, residential rehabilitation, and relapse prevention[2].

Mental excitement, hypersexuality, agitation and violent behavior are common effects of high-dose METH. Long-term use of METH can produce strong dependence and, once the drug is stopped, withdrawal symptoms will appear. During METH dependence withdrawal, patients may experience intense drug cravings, depression, anxiety, fatigue, and mental illness[6]. If the withdrawal symptoms are not well treated, they may cause more severe problems[7]. Increasing evidence showed that METHdependent patients experience impaired cognitive functions (i.e., executive function, attention, social cognition, and working memory)[8]. These deficiencies may partially lead to persistent drug use and poor or unhealthy decision-making [9]. Cognitive defects can also cause behavioral changes, associated with high levels of impulsiveness, hostility and aggression[10,11]. Desey Tziortzis et al. reported a marginal relationship between impulsivity and craving[12], suggesting the degree of impulsivity can positively predict the probability of relapse. Research has also shown that people who abuse METH for a long time often experience depressive symptoms, especially during METH withdrawal[13]. It is common for depression and substance use to occur at the same time[14]. Some researchers support pharmacological approaches to treat METH use or withdrawal and/or depression, while others favour nonpharmacological therapies, such as CBT and mindfulness therapy[15]. The effectiveness of pharmacological interventions to treat METH addiction and the resulting symptoms seems to be limited[16, 17]. Most common intervention strategies still focus on non-pharmacological treatment.

Previous studies suggested that early stressful life events are important risk factors for alcohol and illicit substances addiction[18].However, not all responses to stress are bad, as some stressors may mobilize resilient attempts to protect individuals from adverse factors[19]. Resilience is the flexible use of psychological resources for adapting to adversity [20]. Dyer and McGuinness indicated that resilience describes a process whereby people bounce back from adversity and go on with their lives[21]. It is a dynamic process highly influenced by protective factors. However, some researchers have investigated resilience as an individual trait characterized by hardiness, that is associated with three primary attributes: a greater sense of control over their lives, commitment to the areas of their life even when experiencing stressors, and a perception that change is a challenge rather than a threat[22]. Some studies also highlighted the role of resilience in the prevention of substance addiction. For example, Dullius et al. suggested resilience played an important role in moderating stress and negative emotions in patients with alcohol dependence[23]. And low resilience was reported to be related to alcohol or drug problems and poor working memory performance[24]. Furthermore, a study suggested brain networks in patients with drug addiction showed impaired goal-oriented actions were associated with the resilience system related to behavior regulation[25]. Since mental resilience is the flexible adaptability of dealing with stress-related mental diseases, we sought to explore the connection between resilience and METH addiction during protracted withdrawal to provide psychotherapy suggestions. 
The level of resilience may be related to cognition, depression levels and the degree of impulsivity. The hypothesis assessed during the current study is that the improvement of resilience levels positively affects the improvement of the above factors.

\section{Methods}

\section{Participants}

One hundred and twelve male individuals were recruited from the Huanglong Compulsory Isolated Detoxification Center in Wenzhou between October 2019 and June 2021. The inclusion criteria included: (1) with a history of using METH and fulfill the criteria of Diagnostic and Statistical Manual of Mental Disorders 5th edition (DSM-V) for Stimulant Use Disorder,(2) Han ethnicity, aged between 20 and 45 years old,(3) high school education level above,(3) the score of Wechsler Adult Intelligence test is over 90 points. And the exclusion criteria were:(1) severe diseases such as tumors, serious infections, autoimmune diseases, and other physical disabilities, (2) experiencing other psychotic symptoms, and (3) individuals that refused to participate.

The enrolled individuals were assessed from high to low according to the scores of the Connor-Davidson Resilience Scale. The top $33 \%$ of subjects were assigned to the high resilience group $(n=37)$, the bottom $33 \%$ of the subjects were assigned to the low resilience group $(n=37)$, and the remaining subjects were assigned to the medium resilience group $(n=38)$.

\section{Collection and evaluation of socio-demographic and clinical measures}

The socio-demographic data included age, education, living styles, marital status, religious belief and duration of drug exposure. The clinical measures included the Self-Rating Depression Scale (SDS), the Self-Rating Anxiety Scale (SAS), the Barratt Impulsiveness Scale-11(BIS-11), the Repeatable Battery for the Assessment of Neuropsychological Status (RBANS), the Connor-Davidson Resilience Scale (CD-RISC), and the Somatic Self-Rating Scale (SSS).

SDS is a self-rated scale to evaluate the severity of depression[26], and consists of 20 questions. The standard score is calculated by summing all question scores, and the total score is 1.25 times the standard score, using an integral part. The cut-off point is 50 , and the higher the score correlates to more severe depression.

SAS is also a self-rated scale, which is used to assess the degree of anxiety. It consists of 20 items on a 1-4 Likert-type scale. The cut-off point is 40 , and a higher score suggests more severe anxiety[27].

The BIS-11 scoring consists of 30 items on a four-point Likert scale ( $1=$ "rarely/never" to $4=$ "almost always/always"), which is used to measure impulsivity[28]. The scales can be divided into three dimensions (cognitive, motor, and non-planned impulsivity). The higher scores of each scale suggest the higher impulsivity levels shown by individuals. 
RBANS scoring comprises 12 subtests that yield five index scores (immediate memory, visuospatial, language, attention, and delayed memory) and one total score. The total scale score of RBANS can be used to evaluate cognitive function, and the higher the total scale score, the better the cognitive function[29].

CD-RISC is a self-reported 25-item scale, which is used to evaluate the resilience. The scale employs 5point Likert-type ratings ranging from 0 (not true at all) to 4 (true all the time)[30].

SSS is used to examine the possible emotional problems and corresponding somatization symptoms. The SSS includes 20 items, with each item scored on a 4-point scale (1, none, 2, slight, 3, moderate, and 4, marked). The higher the score, the more severe the somatization symptoms[31, 32].

\section{Statistical analysis}

Normal distribution was assessed by the Shapiro-Wilk test. The socio-demographic and clinical characteristics of the low resilience, moderate resilience and high resilience groups were compared using the one-way analysis of variance (ANOVA) for continuous variables and the chi-square test for categorical variables. Homogeneity of variances test and between-subjects effects test were used. Besides, we also performed post hoc pairwise comparisons. In addition, the correlations among three dimensions of CDRISC and SDS, SAS, BIS-11 and Rbans were also evaluated. Bonferroni corrections were applied to adjust for multiple testing. Further, ordinal regression was used to evaluate the risk factors for resilience during protracted METH withdrawal. All data analysis was calculated with SPSS version 25.0 with two-sided $p$ values of 0.05 .

\section{Results}

The data was normally distributed (the result of Shapiro-Wilk test was listed in supplemental Table 1). The socio-demographics and the clinical characteristics of the three groups were summarized in Table 1. There was no significant difference among the groups in age, education, living styles, marital status, religious belief, the duration of drug exposure as well as the scores of SSS. Further, the main results revealed that high and medium resilience groups showed significantly lower SDS scores compared with the low resilience group (both $p<0.05$ ). Participants with high resilience also reported lower scores of SAS compared with those with low resilience $(p<0.05)$. Notably, the high resilience group showed lower cognitive impulsiveness, non-planning impulsiveness and BIS-11 total scores than low resilience group (all $\mathrm{p}$ <0.05). Besides, compared with low resilience group, the delayed memory was better in high resilience group $(p<0.05)$ and attention was better in the medium resilience group $(p<0.05)$. The total scores of Rbans were also higher in high and medium resilience groups than low resilience group (both $p<0.05$ ). Homogeneity of variances test was checked (all $p>0.05$, Supplemental Table 2 ) and the reliability of the relationship was good (all p>0.05, Supplemental Table 3). 
Table 1

Socio-demographics and clinical characteristics among the groups of low, medium and high resilience.

\begin{tabular}{|c|c|c|c|c|c|}
\hline & $\begin{array}{l}\text { Low } \\
\text { resilience } \\
(n=37)\end{array}$ & $\begin{array}{l}\text { Medium } \\
\text { resilience } \\
(n=38)\end{array}$ & $\begin{array}{l}\text { High } \\
\text { resilience } \\
(n=37)\end{array}$ & $\begin{array}{l}\text { Overall } \\
\text { P-value }\end{array}$ & $\begin{array}{l}\text { Post hoc } \\
\text { comparisons }\end{array}$ \\
\hline Age, mean(SD) & $35.72(5.54)$ & $35.92(6.29)$ & $35.40(5.68)$ & 0.951 & \\
\hline Education & & & & 0.553 & \\
\hline $\begin{array}{l}\text { primary school } \\
\text { education level, n } \\
(\%)\end{array}$ & $5(13 \%)$ & $2(4 \%)$ & $5(13 \%)$ & & \\
\hline $\begin{array}{l}\text { Junior high school } \\
\text { education level, n } \\
(\%)\end{array}$ & $25(67 \%)$ & $25(67 \%)$ & $21(57 \%)$ & & \\
\hline $\begin{array}{l}\text { Senior high school } \\
\text { education level, } n \\
\text { (\%) }\end{array}$ & $7(20 \%)$ & $11(29 \%)$ & $11(30 \%)$ & & \\
\hline Living styles & & & & 0.840 & \\
\hline Live with family & $25(67 \%)$ & $24(64 \%)$ & $25(70 \%)$ & & \\
\hline Live without family & $12(33 \%)$ & $14(36 \%)$ & $11(30 \%)$ & & \\
\hline Marital status & & & & 0.424 & \\
\hline Married & $20(53 \%)$ & $17(44 \%)$ & $16(44 \%)$ & & \\
\hline Not married & $13(34 \%)$ & $11(29 \%)$ & $15(40 \%)$ & & \\
\hline Divorced & $4(13 \%)$ & $10(27 \%)$ & $6(16 \%)$ & & \\
\hline Religious belief & & & & 0.349 & \\
\hline Yes & $21(56 \%)$ & $17(44 \%)$ & $15(40 \%)$ & & \\
\hline No & $16(44 \%)$ & $21(56 \%)$ & $22(60 \%)$ & & \\
\hline $\begin{array}{l}\text { Duration of drug } \\
\text { exposure, mean } \\
\text { (SD) }\end{array}$ & $13.72(6.32)$ & 12.92(6.70) & $14.56(7.52)$ & 0.701 & \\
\hline CD-RISC, mean(SD) & $\begin{array}{l}31.12(10 . \\
41)\end{array}$ & $56.80(6.04)$ & 78.08(7.44) & $<0.001^{\star \star \star *}$ & $\begin{array}{l}1<2 \star \star \star ; \\
1<3^{\star \star \star *} ; 2<3 \star \star \star \star\end{array}$ \\
\hline SDS, mean(SD) & $55.76(7.28)$ & 47.63(9.44) & $40.04(8.48)$ & $<0.001 * \star \star$ & $\begin{array}{l}1>2 \star * ; \\
2>3^{\star *} ; 1>3^{\star \star *}\end{array}$ \\
\hline
\end{tabular}

Note: CD-RISC the Connor-Davidson Resilience Scale; SDS Self-rating depression scale; SAS Self-rating anxiety scale; SSSSomatic Self- rating Scale; BIS-11 Barratt Impulsiveness Scale-11; Rbans The Repeatable Battery for the Assessment of Neuropsychological Status. Post-hoc pairwise comparisons (1= Low resilience; $2=$ Medium resilience;3= High resilience). ${ }^{\star} p<0.05 ;{ }^{* \star} p<0.01 ;{ }^{* \star \star} p<0.001$. 


\begin{tabular}{|c|c|c|c|c|c|}
\hline & $\begin{array}{l}\text { Low } \\
\text { resilience } \\
(\mathrm{n}=37)\end{array}$ & $\begin{array}{l}\text { Medium } \\
\text { resilience } \\
(\mathrm{n}=38)\end{array}$ & $\begin{array}{l}\text { High } \\
\text { resilience } \\
(\mathrm{n}=37)\end{array}$ & $\begin{array}{l}\text { Overall } \\
\text { P-value }\end{array}$ & $\begin{array}{l}\text { Post hoc } \\
\text { comparisons }\end{array}$ \\
\hline SAS, mean(SD) & 42.84(7.39) & $42.60(6.51)$ & $38.28(7.40)$ & $0.045^{\star}$ & $1>3 * ; 2>3^{*}$ \\
\hline SSS, mean(SD) & 28.04(7.99) & $29.40(8.42)$ & $26.44(6.12)$ & 0.388 & \\
\hline \multicolumn{6}{|l|}{ BIS-11 } \\
\hline $\begin{array}{l}\text { cognitive } \\
\text { impulsiveness, } \\
\text { mean(SD) }\end{array}$ & $30.94(5.70)$ & 28.57(3.10) & $26.26(5.04)$ & $0.004^{\star *}$ & $1>3 * \star$ \\
\hline $\begin{array}{l}\text { motor } \\
\text { impulsiveness, } \\
\text { mean(SD) }\end{array}$ & 21.28(8.73) & $24.27(6.40)$ & $20.57(6.85)$ & 0.179 & \\
\hline $\begin{array}{l}\text { non-planning } \\
\text { impulsiveness, } \\
\text { mean(SD) }\end{array}$ & $32.05(7.46)$ & $28.42(6.47)$ & $25.42(8.03)$ & $0.009 * *$ & $1>3 * \star$ \\
\hline $\begin{array}{l}\text { Scale total scores, } \\
\text { mean(SD) }\end{array}$ & $84.28(14.04)$ & $81.27(12.23)$ & $72.27(15.57)$ & $0.01 *$ & $1>3^{\star \star}$ \\
\hline \multicolumn{6}{|l|}{ Rbans } \\
\hline $\begin{array}{l}\text { Immediate Memory, } \\
\text { mean(SD) }\end{array}$ & $72.80(12.21)$ & $75.28(12.18)$ & 78.84(11.59) & 0.209 & \\
\hline $\begin{array}{l}\text { Visuospatial, } \\
\text { mean(SD) }\end{array}$ & 75.79(13.54) & $82.20(14.39)$ & $83.04(15.47)$ & 0.170 & \\
\hline $\begin{array}{l}\text { Attention, } \\
\text { mean(SD) }\end{array}$ & $96.76(12.54)$ & $105.24(13.55)$ & 102.72(17.86) & 0.123 & $1<2^{\star}$ \\
\hline $\begin{array}{l}\text { Language, } \\
\text { mean(SD) }\end{array}$ & 79.28(11.78) & $84.44(13.41)$ & $84.64(13.47)$ & 0.257 & \\
\hline $\begin{array}{l}\text { Delayed memory, } \\
\text { mean(SD) }\end{array}$ & 75.56(14.85) & $82.80(12.78)$ & $85.71(19.40)$ & 0.074 & $1<3^{*}$ \\
\hline $\begin{array}{l}\text { Scale total scores, } \\
\text { mean(SD) }\end{array}$ & 73.64(9.29) & $84.24(10.24)$ & $82.72(11.28)$ & $0.003^{\star \star}$ & $1<2 \star \star ; 1<3 * \star$ \\
\hline \multicolumn{6}{|c|}{ 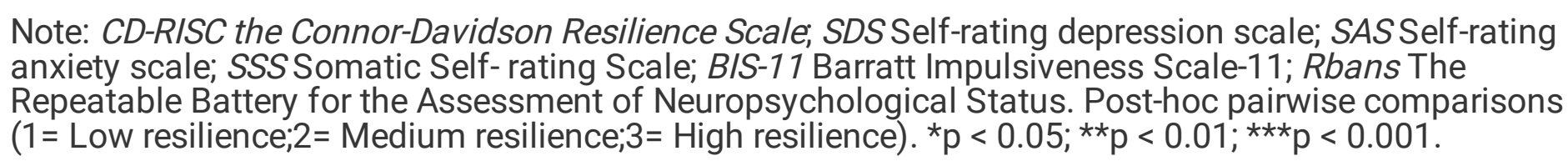 } \\
\hline
\end{tabular}

Table 2 revealed significant negative correlations among the dimensions of hardness, strength, optimism and SDS scores. Additionally, the three dimensions of CD-RISC were also significantly negatively correlated with the BIS-11 scores. These significant differences all passed Bonferroni correction $(\mathrm{p}<$ $0.05 / 12)$. 
Table 2

Inter-correlations among three dimensions of CD-RISC and SDS, SAS, BIS-11 and Rbans.

\begin{tabular}{|lllll|}
\hline & SDS & SAS & BIS-11 & Rbans \\
\hline Hardiness & $-0.539^{*}$ & -0.198 & $-0.279^{*}$ & 0.249 \\
\hline Strength & $-0.620^{*}$ & -0.234 & $-0.296^{*}$ & 0.340 \\
\hline Optimism & $-0.543^{*}$ & -0.210 & $-0.242^{*}$ & 0.304 \\
\hline $\begin{array}{l}\text { Note: } \text { SDS Self-rating depression scale; SAS Self-rating anxiety scale; BIS-11 Barratt Impulsiveness } \\
\text { Scale-11; Rbans The Repeatable Battery for the Assessment of Neuropsychological Status. * } \\
\text { Bonferroni corrected p<0.05/12. }\end{array}$ & \multicolumn{4}{l}{} \\
\hline
\end{tabular}

Table 3 reported the factors which affect resilience during protracted METH withdrawal. And the results showed that the scores of SDS had a significant negative effect on resilience, with an OR of $0.84(p<$ $0.001)$. 
Table 3

Ordinal regression for factors which affect resilience during protracted METH withdrawal.

\begin{tabular}{|c|c|c|c|c|c|c|c|}
\hline \multirow[t]{2}{*}{ Variable } & \multirow[t]{2}{*}{ Parameter } & \multirow[t]{2}{*}{ B } & \multirow[t]{2}{*}{ SE } & \multirow[t]{2}{*}{$\begin{array}{l}\text { Odds } \\
\text { Ratio }\end{array}$} & \multirow[t]{2}{*}{$P$ value } & \multicolumn{2}{|c|}{$\begin{array}{l}95 \% \\
\text { Confidence } \\
\text { Interval }\end{array}$} \\
\hline & & & & & & Lower & Upper \\
\hline \multirow[t]{2}{*}{ Threshold } & Low resilience & -4.102 & 3.178 & - & - & - & - \\
\hline & Medium resilience & -1.917 & 3.146 & - & - & - & - \\
\hline Age & Age & -0.014 & 0.049 & 0.986 & 0.782 & 0.896 & 1.086 \\
\hline Living styles & $\begin{array}{l}\text { Living without family } \\
\text { vs. Living with family }\end{array}$ & -0.048 & 0.529 & 0.953 & 0.928 & 0.338 & 2.690 \\
\hline $\begin{array}{l}\text { Education } \\
\text { years }\end{array}$ & Education years & 0.064 & 0.107 & 1.066 & 0.551 & 0.864 & 1.315 \\
\hline $\begin{array}{l}\text { Duration of } \\
\text { drug exposure }\end{array}$ & $\begin{array}{l}\text { Duration of drug } \\
\text { exposure }\end{array}$ & -0.027 & 0.042 & 0.973 & 0.516 & 0.896 & 1.057 \\
\hline Depression & SDS & -0.170 & 0.043 & 0.843 & $<0.001^{\star \star \star \star}$ & 0.776 & 0.917 \\
\hline Anxiety & SAS & 0.089 & 0.050 & 1.093 & 0.073 & 0.992 & 1.204 \\
\hline Impulsivity & BIS-11 & -0.015 & 0.020 & 0.985 & 0.444 & 0.947 & 1.024 \\
\hline Cognition & Rbans & 0.038 & 0.025 & 1.039 & 0.124 & 0.990 & 1.090 \\
\hline \multicolumn{8}{|c|}{ Notes: Nagelkerke $\mathrm{R}^{2}=48.2 \%$. Model fit $\mathrm{P}<0.001$} \\
\hline \multicolumn{8}{|c|}{$\begin{array}{l}\text { SDS Self-rating depression scale; SAS Self-rating anxiety scale; BIS-11 Barratt Impulsiveness Scale-11; } \\
\text { Rbans The Repeatable Battery for the Assessment of Neuropsychological Status. }\end{array}$} \\
\hline \multicolumn{8}{|c|}{${ }^{\star} \mathrm{p}<0.05 ; * \star \mathrm{p}<0.01 ; * \star \star \mathrm{p}<0.001$} \\
\hline
\end{tabular}

\section{Discussion}

From what we know, this is the first study to investigate the relationships between resilience and impulsivity, cognitive function as well as emotion among METH-dependent patients during protracted METH withdrawal. We primarily found that high levels of resilience are associated with lower degrees of impulsivity and cognitive impairments and a lower level of depression. Early intervention to provide drug addicts with tools to encourage resilience is essential.

Cognitive functions will generally be impaired in patients addicted to drugs $[33,34]$. The cognitive impairment will seriously affect the rehabilitation treatment for METH-dependent patients, especially during withdrawal. Therefore, it is necessary to find a way to improve cognitive deficit in METH dependence patients undergoing withdrawal. This study shows the relationship between high level of 
resilience and good cognitive function in METH dependent patients during protracted withdrawal, which indicated that psychological resilience might enable a breakthrough. Such data infers that METHdependent patients with relatively high resilience may have improved delayed memory and attention. Understanding the impact of mental resilience on cognitive function impairment is relatively limited at this stage, and there is little direct evidence on their relationship. Despite this, the correlation can be broadly understood using the cognitive reserve hypothesis. Cognitive reserve is the ability to maintain cognitive function even if the brain injury occurs [35]. Harmonious relationship can improve cognitive reserve, and continuous positive stimulation can enhance the prevalent development of nerve tissue, promote new nerve pathways generation, and compensate for neurocognitive function impairment[36]. Generally, people with good mental resilience can better deal with situations, creating a supportive psychological environment and promoting cognitive function. In contrast, people with low mental resilience levels cannot quickly restore balance when facing adverse events and exist in a state of chronic stress[37], such as protracted withdrawal. Chronic pressure can induce dendrites of the medial prefrontal cortex and hippocampal vertebral body neurons to retract, thereby causing damage to spatial memory and learning ability $[38,39]$. Additionally, chronic stress can reduce brain-derived neurotrophic factor (BDNF) in the brain, affecting the memory processing by the hippocampus[40].

During early withdrawal, drug abusers will show a high frequency of impulsivity[41]. However, few studies have explored impulsive behaviors during the protracted withdrawal. The current study also found the connection between resilience and impulsivity during the protracted withdrawal. Although impulsivity may be a pre-existing characteristic that makes an individual prone to start taking drugs, it may also be a consequence of drug abuse[42, 43]. Previous METH use may cause changes in the brain, which in turn has a long-term impact on the changes within the typical decision-making process framework[44]. Thus, during the protracted withdrawal, METH users will show varying degrees of impulsivity. Many studies have investigated the relationship between resilience and impulsivity $[45,46]$. The negative correlation between resilience and impulsivity in the period of protracted METH withdrawal may be explained by interconnections between specific neural circuits associated with resilience and impulsivity. The activation of ventromedial prefrontal cortex (vmPFC) has something to do with the promotion of resilience in response to stress[45]. Besides, impulsivity is inversely correlated to gray matter volume in the vmPFC $[46,47]$. Thus, we inferred that long-term effect of METH abuse during the protracted withdrawal may result in abnormal activity or volume in vmPFC. High level of resilience may enhance the repair function of vmPFC and reduce the impulsivity[48].

What's more, the relative higher resilience was associated with lower degree of depression during protracted withdrawal. And the three dimensions of CD-RISC (hardness, strength and optimism) were all negatively related to SDS scores. The result was in line with previous research that resilience is correlated with the symptoms of depression[38] and high scores of mental resilience correspond to lower depressive symptoms[49]. However, the main underlying connection was still unknown. Even though, the possible mechanism can be explained as follows. Wang et al. found that resilience was negatively associated with perceived stress and depression[50]. And several studies suggested that perceived stress was positively correlated to depression in drug users[51,52]. Perceived stress is defined as an individual's cognitive 
assessment of their stress level[53], and effective stress management play a vital role in reducing the degree of depression in drug users[54]. In general, the perceived stress on drug users' depression depends on their processing ability, while resilience is a coping ability, constantly adapting and rebounding in unfavorable environments. Compared with people with poor resilience, those with good resilience tend to feel less stress under challenging environments and are more likely to overcome stressful situations. Furthermore, when adjusted to a stressful environment, the symptoms of depression will naturally diminish. As for METH-dependent patients, we know, symptoms of depression usually coincide with substance use and withdrawal[55]. Besides, the craving for drugs is related to depression[56]. Thus, it's crucial to cultivate an individual's resilience in order to better deal with the symptoms of depression caused by a stressful environment.

Drug addiction is a complex process in which stress plays a crucial role[57]. Addiction and stress responses have a common neurobiological pathway that can be altered by environmental stressors[58]. Even after a long period of abstinence, it is easy to fall back into drug-seeking situations after a stressful experience[59]. Besides, the symptoms appeared in the withdrawal may add difficulties of intervention. Resilience refers to the relative protection of individuals against stress. Therefore, the current study postulated that improving resilience was necessary. For drug addicts, the barriers to resilience may include an imbalance between work and personal life, excessive exposure to stressful events, insufficient time and space to deal with negative emotions, and social isolation[60]. Effective interventions promoting resilience should ideally positively impact one or more of these barrier features, which may be available from the following three strategies. Firstly, training, experience, and perception seem to be essential for enhancing resilience. For example, METH addicts can learn "mindfulness", which focuses on the present mental process, to avoid irrelevant, harmful external stimuli. Secondly, social support appears to be protective. When social support is lacking, METH users may choose to escape and become muffled. Thus, establishing a correct concept of interpersonal communication is an excellent to obtain social support, because it is the basis for establishing good communication between people. Finally, effective coping styles may affect social adaptation in drug addicts, enabling them to become re-engaged with society[61]. For example, teaching stress management for drug addicts. Besides, this study found that the scores of SDS had a significant negative effect on resilience. Another way to increase an individual's resilience level may be to improve the symptoms of depression. All in all, compulsory isolation of drug treatment centers can ensure that drug users are well-trained, thereby improving their adaptability and providing support designed to encourage better coping skills. Some combination of all or some of these strategies will increase the likelihood of more successful rehabilitation of METH-dependent users and may reduce the probability of relapse.

Some limitations of this research to be considered are that the sample size is relatively small and it will be increased in future research. Secondly, this survey was a retrospective study and did not rule out recall errors and observer bias. Thirdly, the causality cannot be confirmed in this research. In the future, we will explore the direction of cause and effect. Fourthly, certain pre-abstinence factors (for example, early stressful life events) which might predict future recovery or deterioration were not evaluated. Finally, only 
male METH-dependent patients were participants, and so female METH-dependent patients will be investigated in the future.

\section{Conclusion}

The current study highlighted the role of resilience during protracted METH withdrawal. Since METH addiction is a severe global public problem, it's necessary to find effective ways to treat the symptoms caused by METH. Psychotherapy has always been used in substance addiction. This study suggested higher resilience is correlated with lower impulsivity levels, better cognitive function and lower depression. For drug addicts, perhaps it may be effectively prevented and well cured by improving resilience.

\section{Abbreviations}

Methamphetamine(METH), The Connor-Davidson Resilience Scale (CD-RISC),Self-rating depression scale(SDS), Self-rating anxiety scale(SAS), Barratt Impulsiveness Scale-11(BIS-11), The Repeatable Battery for the Assessment of Neuropsychological Status(Rbans)

\section{Declarations}

\section{Ethics approval and consent to participate}

All participants signed informed consent documents before participating in the research. This study was performed based on the Helsinki Declaration. The study was approved and monitored by the ethics committee of School of Mental Health, Wenzhou Medical University.

\section{Consent for publication}

Not applicable

\section{Availability of data and materials}

The datasets generated and/or analysed during the current study are not publicly available due to the privacy of participants and because this is a part of the long-term study, but are available from the corresponding author on reasonable request.

\section{Competing interests}

The authors declare that they have no competing interests.

\section{Fundings}

This work was supported by the Science and Technology Program of Wenzhou (S20180012, Y20190098) and National College Students Innovation and Entrepreneurship Training Program (202010343059X). 
$\mathrm{KZ}, \mathrm{HH}, \mathrm{WW}$ and SYZ conceptualized and designed the study. WR, CHP and SYT recruited the participants and completed the screening assessments. LH and WJ analyzed the data and performed the statistical analysis. ZSY, FFC, YLJ, MJL, XPW and MDL wrote the first draft of the manuscript. All authors revised the manuscript and approved the final manuscript.

\section{Acknowledgments}

We are grateful to all participants who made contributions to our study.

\section{References}

1. World Health Organization: World Drug Report 2020. In. Available online at: https://wdr.unodc.org/wdr2020/field/WDR20_Booklet_2.pdf.; 2020.

2. Ballester J, Valentine G, Sofuoglu M: Pharmacological treatments for methamphetamine addiction: current status and future directions. Expert Rev Clin Pharmacol 2017, 10(3):305-314.

3. Huang C, Yuan Q, Zhang L, Wang L, Cui S, Zhang K, Zhou X: Associations Between Childhood Trauma and the Age of First-Time Drug Use in Methamphetamine-Dependent Patients. Front Psychiatry 2021, 12:658205.

4. Chiang M, Lombardi D, Du J, Makrum U, Sitthichai R, Harrington A, Shukair N, Zhao M, Fan X: Methamphetamine-associated psychosis: Clinical presentation, biological basis, and treatment options. Hum Psychopharmacol 2019, 34(5):e2710.

5. Courtney KE, Ray LA: Methamphetamine: an update on epidemiology, pharmacology, clinical phenomenology, and treatment literature. Drug Alcohol Depend 2014, 143:11-21.

6. Mancino MJ, Gentry BW, Feldman Z, Mendelson J, Oliveto A: Characterizing methamphetamine withdrawal in recently abstinent methamphetamine users: a pilot field study. Am J Drug Alcohol Abuse 2011, 37(2):131-136.

7. Newton TF, De La Garza R, 2nd, Kalechstein AD, Tziortzis D, Jacobsen CA: Theories of addiction: methamphetamine users' explanations for continuing drug use and relapse. Am J Addict 2009, 18(4):294-300.

8. Mizoguchi $\mathrm{H}$, Yamada K: Methamphetamine use causes cognitive impairment and altered decisionmaking. Neurochem Int 2019, 124:106-113.

9. Bernheim A, See RE, Reichel CM: Chronic methamphetamine self-administration disrupts cortical control of cognition. Neurosci Biobehav Rev 2016, 69:36-48.

10. Dawe S, Davis P, Lapworth K, McKetin R: Mechanisms underlying aggressive and hostile behavior in amphetamine users. Curr Opin Psychiatry 2009, 22(3):269-273.

11. Plüddemann A, Flisher AJ, McKetin R, Parry C, Lombard C: Methamphetamine use, aggressive behavior and other mental health issues among high-school students in Cape Town, South Africa. 
Drug Alcohol Depend 2010, 109(1-3):14-19.

12. Tziortzis D, Mahoney JJ, 3rd, Kalechstein AD, Newton TF, De La Garza R, 2nd: The relationship between impulsivity and craving in cocaine- and methamphetamine-dependent volunteers. Pharmacol Biochem Behav 2011, 98(2):196-202.

13. Kalechstein AD, Newton TF, Longshore D, Anglin MD, van Gorp WG, Gawin FH: Psychiatric comorbidity of methamphetamine dependence in a forensic sample. J Neuropsychiatry Clin Neurosci 2000, 12(4):480-484.

14. McKetin R, Lubman DI, Lee NM, Ross JE, Slade TN: Major depression among methamphetamine users entering drug treatment programs. Med J Aust 2011, 195(3):S51-55.

15. Hellem TL, Lundberg KJ, Renshaw PF: A review of treatment options for co-occurring methamphetamine use disorders and depression. J Addict Nurs 2015, 26(1):14-23; quiz E11.

16. Chan B, Freeman M, Kondo K, Ayers C, Montgomery J, Paynter R, Kansagara D: Pharmacotherapy for methamphetamine/amphetamine use disorder-a systematic review and meta-analysis. Addiction 2019, 114(12):2122-2136.

17. Siefried KJ, Acheson LS, Lintzeris N, Ezard N: Pharmacological Treatment of Methamphetamine/Amphetamine Dependence: A Systematic Review. CNS Drugs 2020, 34(4):337365.

18. Enoch MA: The role of early life stress as a predictor for alcohol and drug dependence. Psychopharmacology (Berl) 2011, 214(1):17-31.

19. McEwen BS: Protective and damaging effects of stress mediators: central role of the brain. Dialogues Clin Neurosci 2006, 8(4):367-381.

20. Waugh CE, Fredrickson BL, Taylor SF: Adapting to life's slings and arrows: Individual differences in resilience when recovering from an anticipated threat. J Res Pers 2008, 42(4):1031-1046.

21. Dyer JG, McGuinness TM: Resilience: analysis of the concept. Arch Psychiatr Nurs 1996, 10(5):276282.

22. Sutherland JA, Cook L, Stetina P, Hernandez C: Women in substance abuse recovery: measures of resilience and self-differentiation. West J Nurs Res 2009, 31(7):905-922.

23. Dullius A, Fava S, Ribeiro PM, Terra FS: Alcohol consumption/dependence and resilience in older adults with high blood pressure. Rev Lat Am Enfermagem 2018, 26:e3024.

24. Weiland BJ, Nigg JT, Welsh RC, Yau WY, Zubieta JK, Zucker RA, Heitzeg MM: Resiliency in adolescents at high risk for substance abuse: flexible adaptation via subthalamic nucleus and linkage to drinking and drug use in early adulthood. Alcohol Clin Exp Res 2012, 36(8):1355-1364.

25. Ersche KD, Meng C, Ziauddeen H, Stochl J, Williams GB, Bullmore ET, Robbins TW: Brain networks underlying vulnerability and resilience to drug addiction. Proc Natl Acad Sci U S A 2020, 117(26):15253-15261.

26. Zung WW: A SELF-RATING DEPRESSION SCALE. Arch Gen Psychiatry 1965, 12:63-70.

27. Zung WW: A rating instrument for anxiety disorders. Psychosomatics 1971, 12(6):371-379. 
28. Patton JH, Stanford MS, Barratt ES: Factor structure of the Barratt impulsiveness scale. J Clin Psychol 1995, 51(6):768-774.

29. Randolph C, Tierney MC, Mohr E, Chase TN: The Repeatable Battery for the Assessment of Neuropsychological Status (RBANS): preliminary clinical validity. J Clin Exp Neuropsychol 1998, 20(3):310-319.

30. Connor KM, Davidson JR: Development of a new resilience scale: the Connor-Davidson Resilience Scale (CD-RISC). Depress Anxiety 2003, 18(2):76-82.

31. Qi Zhuang MJ-I, Chun-bo LI: Developing of somatic self-rating scale and its reliability and validity. Chin J Behav Med Brain Sci 2010, 19(9):847-849.

32. Xiao-li Li MJ-I, Ben HE.: Clinical application of self-rating somatization symptoms scale in patients with cardiac neurosis. Chin J Misdiagn 2008, 8(20):4798-4799.

33. Baldacchino A, Balfour DJ, Passetti F, Humphris G, Matthews K: Neuropsychological consequences of chronic opioid use: a quantitative review and meta-analysis. Neurosci Biobehav Rev 2012, 36(9):2056-2068.

34. Potvin S, Stavro K, Rizkallah E, Pelletier J: Cocaine and cognition: a systematic quantitative review. J Addict Med 2014, 8(5):368-376.

35. Wook Yoo S, Han CE, Shin JS, Won Seo S, Na DL, Kaiser M, Jeong Y, Seong JK: A Network Flowbased Analysis of Cognitive Reserve in Normal Ageing and Alzheimer's Disease. Sci Rep 2015, 5:10057.

36. Stern Y, Barnes CA, Grady C, Jones RN, Raz N: Brain reserve, cognitive reserve, compensation, and maintenance: operationalization, validity, and mechanisms of cognitive resilience. Neurobiol Aging 2019, 83:124-129.

37. Banerjee Y, Akhras A, Khamis AH, Alsheikh-Ali A, Davis D: Investigating the Relationship Between Resilience, Stress-Coping Strategies, and Learning Approaches to Predict Academic Performance in Undergraduate Medical Students: Protocol for a Proof-of-Concept Study. JMIR Res Protoc 2019, 8(9):e14677.

38. Connor KM, Zhang W: Recent advances in the understanding and treatment of anxiety disorders. Resilience: determinants, measurement, and treatment responsiveness. CNS Spectr 2006, 11(10 Suppl 12):5-12.

39. Conrad CD, Galea LA, Kuroda Y, McEwen BS: Chronic stress impairs rat spatial memory on the Y maze, and this effect is blocked by tianeptine pretreatment. Behav Neurosci 1996, 110(6):13211334.

40. Hariri AR, Goldberg TE, Mattay VS, Kolachana BS, Callicott JH, Egan MF, Weinberger DR: Brainderived neurotrophic factor val66met polymorphism affects human memory-related hippocampal activity and predicts memory performance. J Neurosci 2003, 23(17):6690-6694.

41. Luan X, Ren W, Zhao K, Su H, Shen H, Chen H, Qiu H, He J: High prevalence of depressive symptoms and impulsivity as well as their relationship during subacute methamphetamine withdrawal in chronic abusers. J Clin Neurosci 2017, 39:1-3. 
42. Dalley JW, Everitt BJ, Robbins TW: Impulsivity, compulsivity, and top-down cognitive control. Neuron 2011, 69(4):680-694.

43. Perry JL, Carroll ME: The role of impulsive behavior in drug abuse. Psychopharmacology (Berl) 2008, 200(1):1-26.

44. Scott JC, Woods SP, Matt GE, Meyer RA, Heaton RK, Atkinson JH, Grant I: Neurocognitive effects of methamphetamine: a critical review and meta-analysis. Neuropsychol Rev 2007, 17(3):275-297.

45. Franklin TB, Saab BJ, Mansuy IM: Neural mechanisms of stress resilience and vulnerability. Neuron 2012, 75(5):747-761.

46. Matsuo K, Nicoletti MA, Peluso MA, Hatch JP, Nemoto K, Watanabe Y, Nery FG, Monkul ES, ZuntaSoares $\mathrm{GB}$, Bowden $\mathrm{CL}$ et al: Anterior cingulate volumes associated with trait impulsivity in individuals with bipolar disorder. Bipolar Disord 2009, 11(6):628-636.

47. Schilling C, Kühn S, Romanowski A, Banaschewski T, Barbot A, Barker GJ, Brühl R, Büchel C, Charlet $\mathrm{K}$, Conrod $\mathrm{PJ}$ et al: Common structural correlates of trait impulsiveness and perceptual reasoning in adolescence. Hum Brain Mapp 2013, 34(2):374-383.

48. Liu H, Zhang C, Ji Y, Yang L: Biological and Psychological Perspectives of Resilience: Is It Possible to Improve Stress Resistance? Front Hum Neurosci 2018, 12:326.

49. Hjemdal O, Vogel PA, Solem S, Hagen K, Stiles TC: The relationship between resilience and levels of anxiety, depression, and obsessive-compulsive symptoms in adolescents. Clin Psychol Psychother 2011, 18(4):314-321.

50. Wang L, Xu B, Gu Y, Zhu J, Liang Y: The mediating and moderating effects of resilience on the relationship between perceived stress and depression in heroin addicts. $J$ Community Psycho/ 2019, 47(3):495-503.

51. Scott RM, Hides L, Allen JS, Burke R, Lubman DI: Depressive and anxiety symptomatology in ecstasy users: the relative contribution of genes, trauma, life stress and drug use. Psychopharmacology (Berl) 2010, 209(1):25-36.

52. Strong DR, Brown RA, Sims M, Herman DS, Anderson BJ, Stein MD: Persistence on a stress-challenge task before initiating buprenorphine treatment was associated with successful transition from opioid use to early abstinence. J Addict Med 2012, 6(3):219-225.

53. Cohen S, Kamarck T, Mermelstein R: A global measure of perceived stress. J Health Soc Behav 1983, 24(4):385-396.

54. Habibi Z, Tourani S, Sadeghi H, Abolghasemi A: Effectiveness of stress management skill training on the depression, anxiety and stress levels in drug addicts after drug withdrawal. Int $J$ High Risk Behav Addict 2013, 2(2):82-86.

55. Zweben JE, Cohen JB, Christian D, Galloway GP, Salinardi M, Parent D, Iguchi M: Psychiatric symptoms in methamphetamine users. Am J Addict 2004, 13(2):181-190.

56. Nakama H, Chang L, Cloak C, Jiang C, Alicata D, Haning W: Association between psychiatric symptoms and craving in methamphetamine users. Am J Addict 2008, 17(5):441-446. 
57. Sinha R, Shaham $Y$, Heilig M: Translational and reverse translational research on the role of stress in drug craving and relapse. Psychopharmacology (Berl) 2011, 218(1):69-82.

58. Koob GF: The role of CRF and CRF-related peptides in the dark side of addiction. Brain Res 2010, 1314:3-14.

59. Mantsch JR, Baker DA, Funk D, Lê AD, Shaham Y: Stress-Induced Reinstatement of Drug Seeking: 20 Years of Progress. Neuropsychopharmacology 2016, 41(1):335-356.

60. Mahmoud NN, Rothenberger D: From Burnout to Well-Being: A Focus on Resilience. Clin Colon Rectal Surg 2019, 32(6):415-423.

61. Brooks S, Amlôt R, Rubin GJ, Greenberg N: Psychological resilience and post-traumatic growth in disaster-exposed organisations: overview of the literature. BMJ Mil Health 2020, 166(1):52-56.

\section{Supplementary Files}

This is a list of supplementary files associated with this preprint. Click to download.

- SupplementalTables.docx 